\title{
Combined double-chambered right ventricle with double-chambered left ventricle: a rare anomaly
}

\author{
Trushar Gajjar, Nageswar Rao, Neelam Desai \\ Sri Sathya Sai Institute of Higher Medical Sciences - Prasanthigram, CTVS Department, Prasanthigram, Andhra Pradesh, India
}

Received: September 26, 2016 Accepted: January 07, 2017 Published online: April 17, 2017

\section{ABSTRACT}

Double-chambered right ventricle with double-chambered left ventricle is a very rare congenital anomaly. An 18-year-old female presented with dyspnea, chest pain, and palpitation. Diagnosis was made using transthoracic echocardiography and was confirmed by angiography. Surgical excision of the anomalous muscle bundles in the right ventricular outflow tract along with excision of the fibrous band from the left ventricle was performed. The postoperative course was uneventful and repeated echocardiogram before discharge showed a right ventricular outflow tract gradient of $8 \mathrm{mmHg}$, no gradient across the left ventricular cavity, and improved biventricular functions. The management strategy is dependent on presence of symptom, associated anomalies and type of pathology.

Keywords: Angiography; double-chambered left ventricle; double-chambered ventricle; echocardiography; infundibular stenosis; subaortic stenosis.

Double-chambered ventricle is a rare congenital cardiac anomaly, where the ventricular chamber is wholly or partially partitioned usually by abnormal muscular ridges or fibrosis. ${ }^{[1-9]}$ Unlike double-chambered right ventricle (DCRV), division of the left ventricle $(\mathrm{LV})$ is a rare anomaly. ${ }^{[1-5]}$ Previously, the description of the double-chambered left ventricle (DCLV) was given by Gerlis et al. ${ }^{[1]}$ and Kay et a1. ${ }^{[2]}$

Herein, we describe a case of DCRV and DCLV in an 18-year-old female.

\section{CASE REPORT}

An 18-year-old female patient presented with dyspnea, chest pain, and palpitation. The physical examination revealed a Grade 4/6 ejection systolic murmur at the left upper sternal border. Chest X-ray showed a cardiothoracic ratio of $50 \%$ with reduced pulmonary blood flow. Electrocardiogram showed right ventricular hypertrophy. Transthoracic echocardiography revealed levocardia and DCRV with a gradient of $70 / 58 \mathrm{mmHg}$ associated with a ledge of muscle tissue in the LV cavity below the mitral valve (Figure 1a) with $30 \mathrm{mmHg}$ gradient across the constriction with figure of 8 appearance in apical 4 chamber view. No regional wall motion abnormality and improved biventricular functions were observed. Cardiac catheterization revealed a right atrial pressure of $21 / 0 \mathrm{mmHg}$ with a mean of $10 \mathrm{mmHg}$, a right ventricular pressure of $157 / 10 \mathrm{mmHg}$, a right ventricular outflow tract of $31 / 10 \mathrm{mmHg}$, a pulmonary artery of $33 / 11 \mathrm{mmHg}$, a pulmonary artery wedge of $17 / 6 \mathrm{mmHg}$, a $\mathrm{LV}$ apex of $162 / 11 \mathrm{mmHg}$, a LV outflow tract of 152/76 $\mathrm{mmHg}$, an aortic pressure of $141 / 83 \mathrm{mmHg}$, and a femoral artery pressure of $157 / 77 \mathrm{mmHg}$. The gradient between the right ventricular body and the outflow tract was $126 \mathrm{mmHg}$, while the gradient across the fibrous ridge between the LV apex and outflow was $10 \mathrm{mmHg}$. Left ventricular angiography showed a ledge of tissue in the mid-LV cavity below the mitral valve (Figure 1b), separating the $L V$ with a hypertrophied distal chamber and thin-walled proximal chamber. Right ventricular angiography revealed the presence of anomalous septal and parietal bands, producing DCRV with a good-sized main pulmonary artery and confluent branch pulmonary arteries. The surgical procedure was performed through a conventional median sternotomy and cardiopulmonary bypass was established by aortic

Corresponding author: Trushar Gajjar, MD. Sri Sathya Sai Institute of Higher Medical Sciences - Prasanthigram, CTVS Department, Prasanthigram, 515134 Andhra Pradesh, India.

Tel: +91 9700355411 e-mail: trushargajjar@gmail.com 

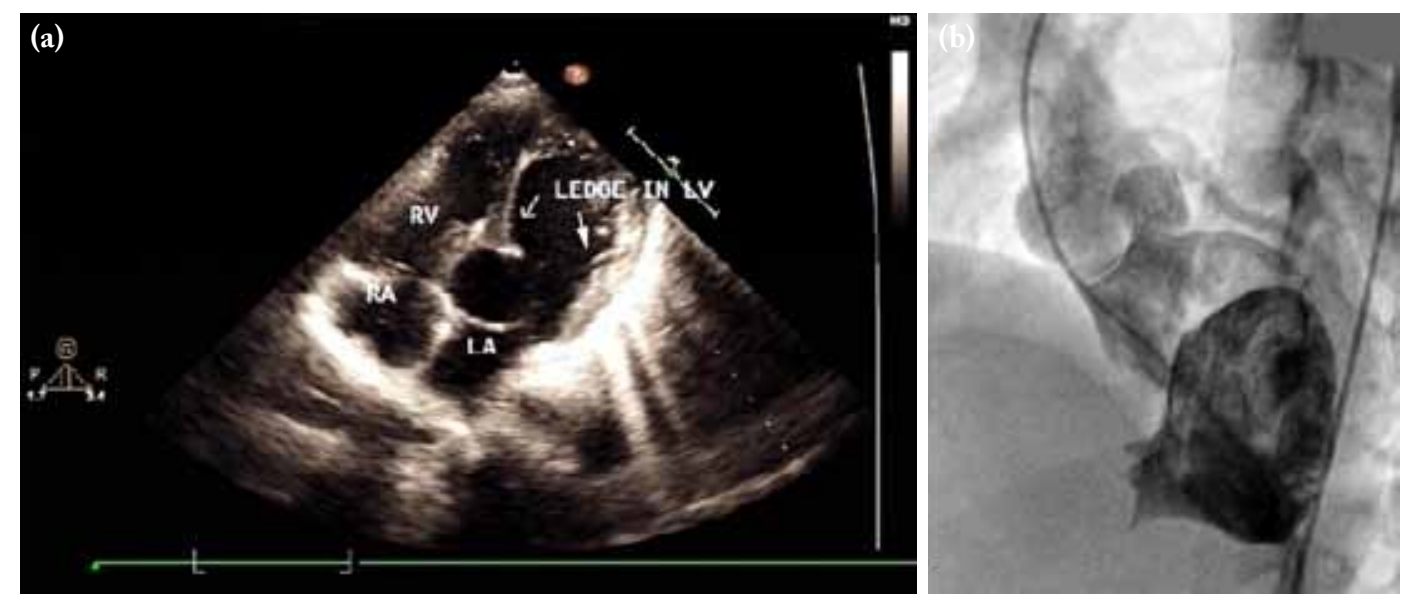

Figure 1. A preoperative echocardiogram and angiogram view. (a) Transthoracic echocardiogram showing ledge of tissue from both the wall of the left ventricle, more from the septal wall, producing double-chambered left ventricle. (b) Left ventriculography showing double-chambered left ventricle with possibly closed apical ventricular septal defect.

RA: Right atrium; RV: Right ventricle; LA: Left atrium; LV: Left ventricle.

and bicaval cannulation. The myocardial protection was provided with cold blood antegrade cardioplegia. After cross-clamping the aorta, the right and the left atrium were opened, and the anatomy was visualized. The intraoperative findings showed a muscular band in the LV cavity below the mitral valve (Figure $2 \mathrm{a}$ ), and there was a tight fibrous ring with anomalous septal and parietal bands in the right ventricular cavity. The ledge of the muscular tissue in the LV cavity was accessed and excised through a left atrial-mitral valve route, and the fibrous ring and muscle bundles in the right ventricle was accessed and excised through the right atrial-tricuspid valve route. Weaning from the cardiopulmonary bypass was performed in a usual fashion. Intraoperative transesophageal echocardiography showed no gradient across the mid LV cavity, trivial mitral regurgitation, and an- $8-\mathrm{mmHg}$ gradient across the right ventricular outflow tract with improved biventricular functions. The postoperative course was uneventful, and transthoracic echocardiography before discharge revealed a widely opened right ventricular outflow tract and mid-LV cavity (Figure 2b) with a right ventricular outflow gradient of $8 \mathrm{mmHg}$ and no gradient in the mid-cavity of the LV with improved biventricular functions. The histopathology examination of the resected tissue from the LV cavity showed a fibrous tissue with normal myocardium.
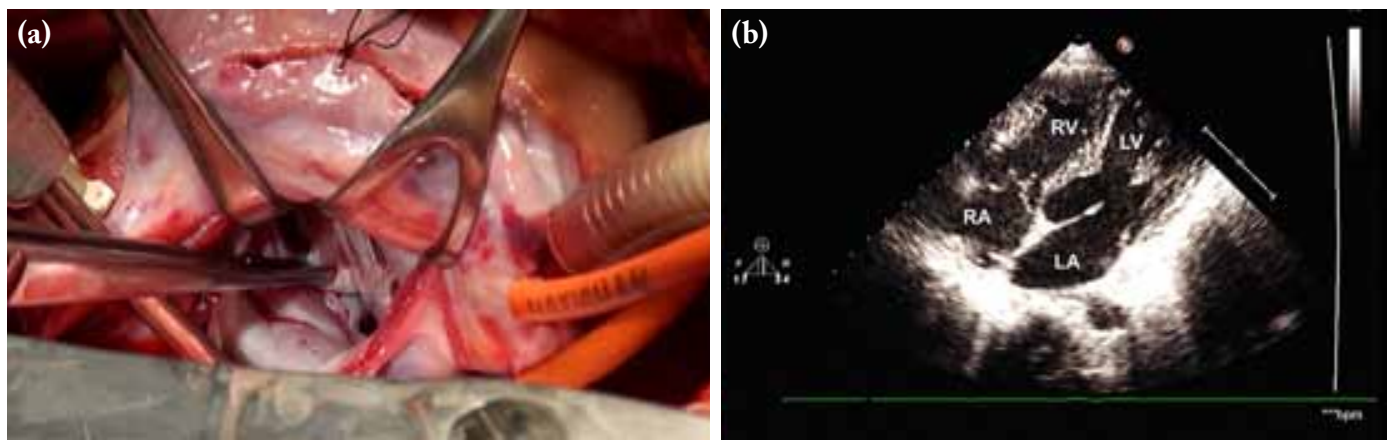

Figure 2. Surgical procedure image and post operative echocardiogram showing. (a) An intraoperative view obtained from the surgeon's side, showing muscle tissue in the left ventricle with fibrosis over, producing double-chambered ventricle. (b) A postoperative transthoracic echocardiogram view showing a widely opened left ventricular cavity.

RA: Right atrium; RV: Right ventricle; LA: Left atrium; LV: Left ventricle. 


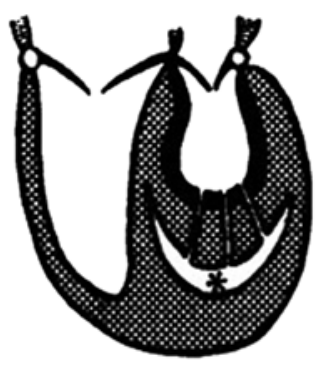

Type 1

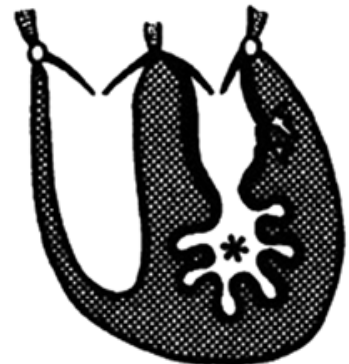

Type 2

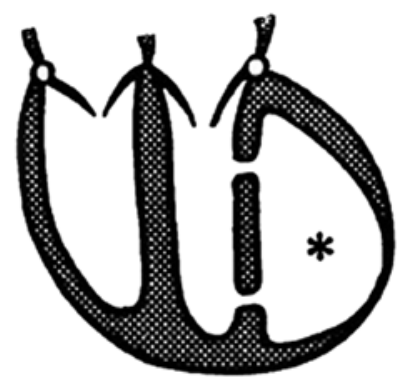

Type 3

Figure 3. Types of double-chambered left ventricle.

Type 1: The left ventricular cavity is divided into two chambers and both the chambers communicate through multiple orifices between the smooth-walled chamber and the left ventricle proper.

Type 2: The left ventricular cavity is divided by the hypertrophied muscular wall with an apical portion having prominent and enlarged branching sinusoids lined by thickened endocardium, more commonly seen with endocardial fibroelastosis.

Type 3: The left ventricle is completely divided into two portions and the both chambers lie side by side.

\section{DISCUSSION}

Double-chambered left ventricle is a very rare congenital cardiac anomaly. ${ }^{[1]}$ This pathology is rarer than DCRV, as described by Kay et al. ${ }^{[2]}$ In addition, the association of DCLV with DCRV is extremely rare. Although the etiology of DCLV is less known, it is thought to be congenital and non-progressive..$^{[1,2,4,5]}$ Various theories for DCLV have been suggested, including post-inflammatory pathologies, defects or hypoplasia of the myocardial wall, and division by a viable myocardium with fibrous opening and the endocardial fibroelastosis. ${ }^{[1,2,4,5]}$ Embryologically, DCLV is caused by the failure of regression of the fetal trabeculations. ${ }^{[4]}$ Gerlis et al. ${ }^{[1]}$ classified DCLV into three types (Figure 3): Type 1, where the LV cavity is divided into two chambers and both chambers communicate through multiple orifices between the smooth-walled chamber and the left ventricle proper. In type 2 , the $\mathrm{LV}$ cavity is divided by the hypertrophied muscular wall with an apical portion, having prominent and enlarged branching sinusoids lined by thickened endocardium, more commonly seen with endocardial fibroelastosis. In type 3 , the $\mathrm{LV}$ is completely divided into two portions and both chambers lie side by side.

Double-chamberedleftventriclecanbedifferentiated from the relatively more common congenital aneurysm and diverticula. ${ }^{[1]}$ It is best differentiated from LV aneurysms and pseudoaneurysms, by the fact that the double-chambered ventricles exhibit normal contractile motions during systole. ${ }^{[1]}$ Ventricular aneurysms also lack complete layering of the ventricular wall, thereby, expanding slightly due to the increased pressure during systole. ${ }^{[1]}$

In general, DCLV is incidentally detected in the course of an evaluation for other cardiovascular abnormalities. The differentiation between DCRV and DCLV is evident, as both have a distinct pathophysiology. The former is more common and often presents with murmur and exertional dyspnea. Several studies have found that DCRV is associated with septal defects, tetralogy of Fallot, and transposition of the great arteries. ${ }^{[5-7]}$ Conversely, the latter one is commonly asymptomatic. In addition, DCRV is often caused by a progressive thickening of the right ventricular septum due to the presence of anomalous muscle bundles, which results in a pressure gradient and formation of two chambers in series. In contrast, the chambers of a DCLV are in parallel and present less of a pressure gradient, as both contract synchronously. ${ }^{[4,6,7]}$ Similarly, in our case, echocardiographic gradient was $30 \mathrm{mmHg}$; however, catheterization gradient was only $10 \mathrm{mmHg}$. The DCLV etiology is less well-known, although the anomaly is thought to be congenital and nonprogressive. As this is an extremely rare finding, no definite data regarding the prognosis, outcomes, and potential complications, such as risk of an embolism, in the DCLV are available. It is often believed that 
DCLV poses little risks to the patient, and treatment is usually guided by the presence of other associated abnormalities. ${ }^{[2,5]}$

Furthermore, the transthoracic and transesophageal echocardiography and computed tomography can aid in the detection of the double-chambered ventricles. ${ }^{[2-5]}$ Transthoracic echocardiography in short-axis inverted views and apical four-chamber views show a transverse muscle band traversing the LV dividing the LV into two halves, producing a typical "figure-of-eight" appearance, indicating a double-chambered. ${ }^{[8]}$ However, cardiac magnetic resonance imaging allows an improved delineation of this condition, thanks to its higher spatial resolution and the ability for tissue characterization, particularly regarding the differentiation between fibrosis and normal myocardium, which would not be easily achievable with echocardiography ${ }^{[8]}$ or computed tomography. ${ }^{[3]}$

In the literature, several treatment strategies have been described. ${ }^{[2,4,5]}$ In asymptomatic patients without any associated anomaly or non-significant gradient across the DCLV, clinical observation and follow-up are recommended. However, in symptomatic cases with or without gradient across the DCLV or with an associated lesion, surgical excision is indicated. There are several surgical techniques in the literature..$^{[2,4,5]}$ For type 1 and type 2 cases, excision of the obstructive shelf is recommended, while accessory chamber exclusion is indicated for type 3 disease. Due to the rarity of DCLV, on the other hand, few data exist on the treatment approaches and outcomes. Surgical excision of the accessory chamber with interposition patch reconstruction and cardiac transplant are two valid options. ${ }^{[4,5]}$

Although our case did not fit to any of the types described by Gerlis et al., ${ }^{1}$ it had a muscular self-like projections producing DCLV which was associated with DCRV. As it was associated with DCRV, presentation was delayed and required excision of an abnormal muscle bridge in the LV cavity along with coring out of the right ventricular cavity. The histopathological examination of the resected tissue showed a fibrous tissue with normal myocardium and no evidence of endocardial fibroelastosis. The etiology probably might be abnormal muscular hypertrophy or poor regression of the fetal trabeculations.
In conclusion, double-chambered right ventricle is a rare congenital anomaly; however, the association of this pathology with double-chambered left ventricle is extremely rare. As in our case, both anomalies are responsible for the clinical presentation. The diagnosis can be achieved by transthoracic echocardiography and can be confirmed by angiography and cardiac magnetic resonance imaging. The treatment modalities vary according to symptoms, associated lesions, and type of the pathology.

\section{Declaration of conflicting interests}

The authors declared no conflicts of interest with respect to the authorship and/or publication of this article.

\section{Funding}

The authors received no financial support for the research and/or authorship of this article.

\section{REFERENCES}

1. Gerlis LM, Partridge JB, Fiddler GI, Williams G, Scott O. Two chambered left ventricle. Three new varieties. Br Heart J 1981;46:278-84.

2. Kay PH, Rigby M, Mulholland HC. Congenital double chambered left ventricle treated by exclusion of accessory chamber. Br Heart J 1983;49:195-8.

3. Deshpande J, Sivaraman A, Vaideeswar P. Double chambered left ventricle. Cardiol Young 1994;4:415-6.

4. Kilner PJ, Geva T, Kaemmerer H, Trindade PT, Schwitter J, Webb GD. Recommendations for cardiovascular magnetic resonance in adults with congenital heart disease from the respective working groups of the European Society of Cardiology. Eur Heart J 2010;31:794-805.

5. Akdemir R, Balci MM, Ozer C, Karcaaltincaba M, Acikel S. Quadrichambered ventricles. Cardiol J 2010;17:303-5.

6. Choi YJ, Park SW Characteristics of double-chambered right ventricle in adult patients. Korean J Intern Med 2010;25:147-53.

7. Truong QA, Yared K, Maurovich-Horvat P, Siegel E, Cubeddu RJ, King ME, et al. Images in cardiovascular medicine. Double-chambered right ventricle and situs inversus with dextrocardia. Circulation 2010;121:229-32.

8. Nacif MS, Mello RA, Lacerda Junior OO, Sibley CT, Machado RA, Marchiori E. Double-chambered left ventricle in an adult: diagnosis by CMRI. Clinics (Sao Paulo) 2010;65:1393-5.

9. Kumar GR, Vaideswar P, Agrawal N, Khandeparkar J, Khandekar J, Patwardhan A. Double chambered ventricles: A retrospective clinicopathological study. Ind J Thorac Cardiovasc Surg 2007;23:135-40. 